\title{
Detection of Mitochondrial Mutations in Cancer by Next Generation Sequencing
}

Fei Ye, David C, Samuels and Yan Guo*

Department of Cancer Biology, Vanderbilt University, USA

“Corresponding author: Yan Guo, Assistant Professor, Department of Cancer Biology, Vanderbilt University, USA, Tel: 615936-0816, E-mail: yan.guo@vanderbilt.edu Rec date: Jan 21, 2014, Acc date: Apr 26, 2014, Pub date: Apr 28, 2014

Copyright:@ 2014 Ye et al. This is an open-access article distributed under the terms of the Creative Commons Attribution License, which permits unrestricted use, distribution, and reproduction in any medium, provided the original author and source are credited.

\begin{abstract}
Mitochondria play a central role in the regulation of cellular function, metabolism, and cell death in many types of cell including cancer. Little attention has been paid to the potential mutations that can affect mitochondrial function in cancer, other than specific mutations in genes that are encoded in the nuclear DNA and so once mutated, all mitochondria in a cell will be affected. However, both somatic and germline mutations in mitochondrial genes are common and have been suggested to contribute to the development of cancer. Recent advances in high-throughput technologies have allowed for the rapid and accurate detection of mitochondrial DNA (mtDNA) mutations, polymorphisms, or copy number variations in a variety of tissues and bodily fluids, and can be used as a tool for early detection and treatment of cancer. In addition to deep-sequencing specifically targeted at mtDNA, mtDNA sequences can also be extracted from exome (or whole genome) sequencing data.
\end{abstract}

\section{Keywords Mitochondria; Cancer; Carcinoma}

\section{Mitochondrial and Cancer}

Mitochondria generate the majority of cellular energy through oxidative phosphorylation, which produces ATP. Mitochondrial dysfunctions are an important cause of many neurological diseases [1] and drug toxicities [2,3] and may contribute to carcinogenesis and tumor progression $[4,5]$ yet their role in tumorigenesis remains largely unknown. Early in the 1930s, Warburg has already discovered that cancer cells rely heavily on glycolysis to meet their metabolic demands (i.e., "aerobic-glycolysis") [6], and several hypothetical mechanisms have been proposed to explain this phenomenon [7]. In 1998, somatic homoplasmic mutations in tumor mtDNA were found that were not present in matched control tissues [8]. Since then, various studies have shown that mutations in mtDNA can contribute to cancer etiology $[9,10]$ and mtDNA mutations are associated with various types of cancer, including breast cancer [11], prostate cancer [12,13], head and neck cancer [14], colon cancer [15], and bladder cancer [16,17]. It is likely that hereditary mitochondrial DNA variations may predispose individuals to cancer, while somatic mutations may possibly affect tumor progression. The functional consequences of mitochondrial mutations largely result in metabolic alteration [18-21] and changes in the protein composition of the mitochondrial inner membrane.

In breast cancer mutations in mitochondrial DNA have been linked to increased breast cancer risk through a deficiency in Electron Transport Chain (ETC) function and altered Reactive Oxygen Species (ROS) levels [22]. Altered expression levels of the Oxidative Phosphorylation System (OXPHOS) subunits or mitochondrial structural injury can impair ATP content and occur in breastInfiltrating Ductal Carcinoma (IDC) [23]. Variations in the mtDNA copy number may be the overall result of gene (hereditary) and environmental interactions (oxidative stress) caused by potential exogenous cancer risk factors such as age, hormones, diet and environmental oxidants/antioxidants, and reaction to oxidative damage [24-26]. While it is difficult to prove the involvement of
mtDNA mutations in triggering oncogenesis, there is increasing evidence that mutated mtDNA is a marker of poor survival in cancer prognosis. The first suggestions that mtDNA mutations may play a role in metastasis came from the comparison of frequency of somatic mtDNA mutations in Non-Small-Cell Lung Cancer (NSCLC) at different stages of tumor formation, which show a significantly decreased survival among advanced NSCLC patients harboring mtDNA mutations. However, very few studies have investigated the role of mtDNA copy number in breast cancer patients and those studies have generated somewhat conflicting results. Researchers have found that both increased mtDNA copy number from whole blood DNA [27] and reduced mtDNA copy number in tissue may increase breast cancer risk [28-30]. This understudied area needs further exploration.

\section{mtDNA Sequencing}

The number of mitochondria in a cell varies greatly by organism and tissue type. Usually, there are about 100 mitochondria in each mammalian cell, and 2-10 copies of mtDNA can be harbored in each mitochondrion [31]. Thus, heteroplasmy is a unique feature often observed with mtDNA mutations. A mtDNA mutation is heteroplasmic, if it is a mixture of normal and mutant mtDNA copies within a cell $[32,33]$. It has been found that heteroplasmies affecting the entire mitochondrial genome are common in normal individuals and moreover, the frequency of heteroplasmic variants varied considerably between different tissues in the same individual, although some of these mutations might have been germline and not somatic [34]. Researchers have found that heteroplasmy could promote tumor growth and that it is closely associated with aging [35-37]. Some studies even suggested that an mtDNA mutation does not need to reach homoplasmy, i.e. all copies of mtDNA within a cell are mutated, to promote tumor growth [24,25] Although this claim still lack definitive proof.

In many earlier studies on mtDNA mutations, data were obtained by Sanger sequencing, which cannot detect low abundant 
heteroplasmies. For more than ten years, a growing number of articles have described somatic mutations of the mitochondrial genome (mtDNA) in human tumors, identified by comparing tumor mtDNA and the mtDNA in adjacent normal tissue or blood based on Sanger sequencing. With the precise quantification achievable with deep sequencing, researchers can determine whether the cancer-specific somatic mutations are heteroplasmic rather than homoplasmic. Previously the sequencing accuracy was a concern in assessing the frequency of mutations in mitochondrial DNA, especially in tumor samples. By tagging both strands of duplex DNA, a recently proposed method called Duplex Sequencing may pave the way to explore the full power of high depth sequencing in answering fundamental questions, through its application to investigating the roles of mitochondria in cancer [38].

The short read sequencing market has been dominated by Illumina's sequencing platform. The most popular platform is HiSeq 2000/2500 which can output up to 200 million reads per sample. Because mitochondrion is very short, roughly 1 million 50 base pair long reads can achieve average depth of 3000x. Usually, multiplexing and barcoding technique will be used in association with mitochondria sequencing. Individual barcode (4 to 8 nucleotides) sequences are added to each sample so they can be distinguished after pooling. On a single lane in HiSeq 2500, up to 96 samples can be pooled together. Illumina's MiSeq platform offers longer read upto 300 bases pairs which allows for better de novo assembly and better detection of structural variants. But MiSeq's throughput is not comparable to HiSeq.

Other than performing high throughput sequencing targeted at mtDNA, mtDNA sequences can also be extracted from other sources of sequencing data. Because of the abundance of mtDNA, mtDNA sequence is one of the most common by product of sequencing data such as exome, whole genome and RNAseq [39]. In exome sequencing data, due to the lack of exome capture efficiency, a small portion of the reads will align to the mitochondrial genome, even when the mitochondrial genome is not the intended sequencing target. Although, the mitochondrial genome composed of almost all coding sequence which fits the definition of exome, however it is usually not included in the part of target DNA for the exome sequencing capture methods commonly used today. Recently, a study has shown that mitochondrial sequences can be extracted from exome sequencing data [40]. The average coverage of the mitochondrial genome from exome sequencing can be as high as several hundred (Figure 1), easily surpassing the average coverage of even the targeted exome regions [41]. The relatively high coverage of mtDNA is caused by the high copy number of mtDNA per cell. The advance of high-throughput sequencing technologies and the typically high coverage of a mtDNA sequence obtained provides a powerful tool for the study of mitochondrial DNA heteroplasmy in unprecedented detail $[32,33,42-44]$. This should be contrasted to techniques that specifically target the mitochondrial sequence, which can produce an average depth of tens of thousands of reads across the mitochondrial genome $[34,42,45,46]$. For mitochondria targeted sequencing, heteroplamsy as few as 0.1 percent are detectable [47]. For mitochondria sequences extracted from exome sequencing data, typically, heterplasmy of $1 \%$ is detectable.

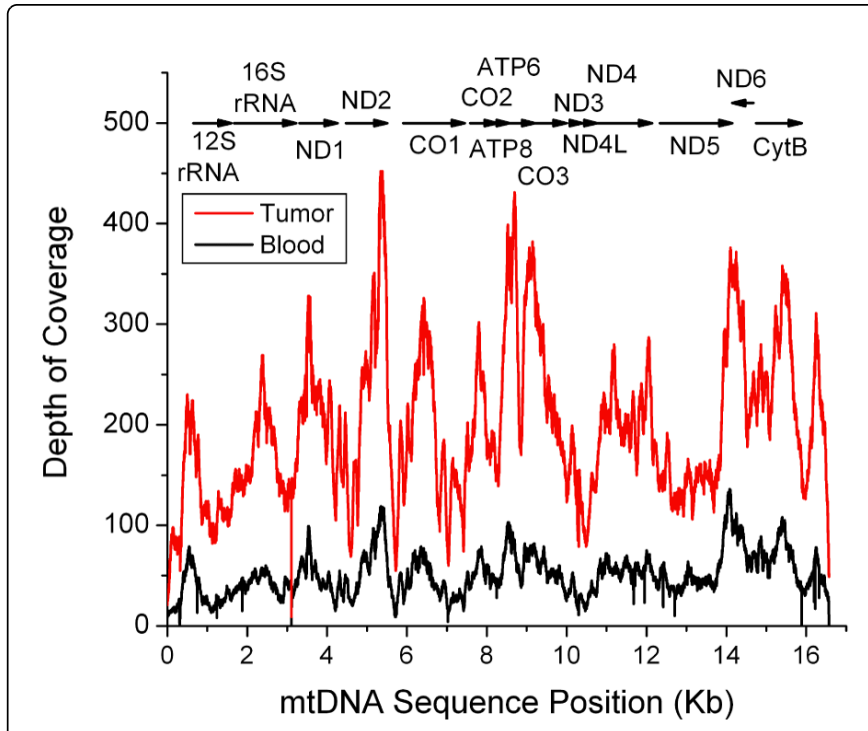

Figure 1: Example of mitochondria sequence depth in exome sequencing data. The red color denotes tumor and the black color denotes blood. The tumor tissue has more copies of mitochondria which resulted higher sequencing depth.

Multiple studies have started to infer mitochondria mutation information from exome sequencing data. The best example is The Cancer Genome Atlas (TCGA) project which contain thousands of exome sequencing data across 20 different types of cancers. Many study cohorts in TCGA have inferred mtDNA somatic mutation from exome sequencing data. Based on the recently published TCGA breast cancer marker paper [48], 325 mitochondrial DNA somatic mutations derived from off-target reads from the 776 exome sequencing data were reported. The concept of extracting mtDNA sequences from exome sequencing data can be easily applied to RNAseq and other type of targeted sequencing data.

\section{mt DNA Mutation Detection by Sequencing}

Sequencing alignment often suffers from ambiguity caused by homologous regions. This problem is amplified with mitochondria sequencing alignment due to the presence of nuclear copies of the mitochondrial genomes (nuMTS) [49,50]. As discussed previously, mitochondria sequences can be a byproduct of other sequencing data, nuclear DNA sequences can also be the byproduct of targeted mitochondria sequencing data. A read sequenced from nuMTS or mtDNA can cause ambiguity about whether it should align to the nuclear or the mitochondrial genome. There are three approaches dealing with the nuMTS ambiguity ranging from tolerant to conservative and simple to complex.

The most tolerant and simplest approach is to align the raw sequence reads against the mitochondrial reference genome directly and then throw out the non-aligned reads. This approach is simple because it ignores the nuMTS in the alignment and it is tolerant because it allows more reads to be aligned. However, this approach also allows nuMTS reads to be aligned to mitochondria genome which nuMTS introduce false heteroplasmic variability in the reported mtDNA sequence. The most conservative and complex approach is to first aligning reads against the nuclear genome only and then align the 
non-aligned reads to the mitochondrial genome. This approach is most conservative because it will allow mtDNA reads align to nuMTS which resulting less reads aligned to mitochondria genome and it is the most complex because it requires two alignment steps at the cost of additional alignment time and data storage.

A compromised approach is to align the raw reads against both the nuclear and mitochondrial genomes simultaneously. It is possible that a read can have two best mapping options, one at nuMTS and one at mitochondria genome. When such scenario happens, we are relied on the aligner used to make the correct decision. Most aligner such as BWA [51] and Bowtie [52] have the options to let the user to choose if this read is counted as 1 mapped randomly in nuMTS and mitochondria genome or counted as a fraction in all of the best mapping matches. The compromised approach has the best balanced between time consumption and misalignment rate. Mito Seek [53] software developed to extract mitochondria sequences from exome sequencing data implemented this approach.

Mitochondrial DNA copy number is highly variable and has been suggested to be associated with many diseases including cancer [27-30]. The traditional methods for assessing mtDNA copy number usually involve PCR [54] technology. A recently a novel method for evaluating mtDNA copy number was developed based on both sequencing-based assay of mitochondria NDA copy number and techniques developed for RNA expression profiling [55].

Mitochondria copy number can also be estimated from exome sequencing data. For example, it has been shown that the fraction of captured mitochondrial sequences in exome sequencing data is proportionalto the relative abundance of the corresponding mitochondrialgenome in the original total DNA extract [22,38,41]. The mtDNA copy extracted from exome sequencing data can be useful when studying tumor samples for conducting association tests with phenotypes such as the tumor stage and metastasis stage. MitoSeek can be used to compute relative mtDNA copy number from exome sequencing data.

\section{Conclusions and Future Prospects}

Mutations of the mtDNA, heteroplasmic in most cases, were detected in different types of cancers, often paired with bodily fluids samples. Studies have also shown mtDNA mutations associated with cancer recurrence [56], genotoxic damage [57], and poor survival in late-stage cancer patients [58,59]. However, despite their high frequency in most solid human tumor types investigated so far, a causal role for a significant portion of mtDNA mutations in cancer cannot be claimed. Although some mtDNA mutations may enhance tumor growth, the enrichment of mutated mtDNA in solid human tumors can be explained by random drift, without need of any selective advantage [60]. More than 30 years after Sanger and colleagues first sequenced human mitochondrial genome, with the power of high depth sequencing technology, we are now able to study the role of mtDNA mutations in cancer to an extent not possible before, especially for off-target mtDNA heteroplasmy. Extending and improving the use of high depth sequencing is thus connected with great expectations to reject or retain current hypotheses proposed to explain the Warburg effect [7]. Furthermore, by assessing mitochondrial DNA content, exome sequencing data has also been used to diagnose certain mitochondrial disorders [61]. Additionally, high depth mtDNA sequencing may bring valuable insights to investigate if mtDNA mutations are associated with RNA-seq gene expression profiles in cellular pathways that regulate mitochondrial DNA synthesis and that are either directly or indirectly targeted by the tested anticancer drugs. MtDNA-dependent gene expression profiles can be identified to determine whether they reflect possible acquired mutations in the nuclear genome and to further investigate possible relationships between mitochondrial activity and gene expression changes, in both normal and cancer cells.

\section{Acknowledgement}

We would like to thank Margot Bjoring for her editorial support. Yan Guo, and Fei Ye were supported by grant CCSG (P30 CA068485).

\section{References}

1. Fernandez-Vizarra E, Bugiani M, Goffrini P, Carrara F, Farina L, et al. (2007) Impaired complex III assembly associated with BCS1L gene mutations in isolated mitochondrial encephalopathy. Hum Mol Genet 16: 1241-1252.

2. Lemasters JJ, Qian T, Bradham CA, Brenner DA, Cascio WE, et al. (1999) Mitochondrial dysfunction in the pathogenesis of necrotic and apoptotic cell death. J Bioenerg Biomembr 31: 305-319.

3. Wallace KB, Starkov AA (2000) mitochondrial targets of drug toxicity. Annu Rev Pharmacol Toxicol 40: 353-388.

4. Modica-Napolitano JS, Singh KK (2004) Mitochondrial dysfunction in cancer. Mitochondrion 4: 755-762.

5. Chen EI (2012) Mitochondrial dysfunction and cancer metastasis. J Bioenerg Biomembr 44: 619-622.

6. Kaiser-Wilhelm-Institutfu $\backslash \mathrm{r}$ Biologie B-D, Warburg OH (1930) The metabolism of tumours : investigations from the Kaiser Wilhelm Institute for Biology, Berlin-Dahlem.

7. Kroemer G (2006) Mitochondria in cancer. Oncogene 25: 4630-4632.

8. Polyak K, Li Y, Zhu H, Lengauer C, Willson JK, et al. (1998) Somatic mutations of the mitochondrial genome in human colorectal tumours. Nat Genet 20: 291-293.

9. Baysal BE, Ferrell RE, Willett-Brozick JE, Lawrence EC, Myssiorek D, et al. (2000) Mutations in SDHD, a mitochondrial complex II gene, in hereditary paraganglioma. Science 287: 848-851.

10. Vanharanta S, Buchta M, McWhinney SR (2004) Early-onset renal cell carcinoma as a novel extraparaganglial component of SDHB-associated heritable paraganglioma. American journal of human genetics 74:153-159.

11. Canter JA, Kallianpur AR, Parl FF, Millikan RC (2005) Mitochondrial DNA G10398A polymorphism and invasive breast cancer in AfricanAmerican women. Cancer Res 65: 8028-8033.

12. Herrmann PC, Gillespie JW, Charboneau L, Bichsel VE, Paweletz CP, et al. (2003) Mitochondrial proteome: altered cytochrome c oxidase subunit levels in prostate cancer. Proteomics 3: 1801-1810.

13. Petrosillo G, Di Venosa N, Ruggiero FM, Pistolese M, D'Agostino D, et al. (2005) Mitochondrial dysfunction associated with cardiac ischemia/ reperfusion can be attenuated by oxygen tension control. Role of oxygenfree radicals and cardiolipin. Biochim Biophys Acta 1710: 78-86.

14. Sun W, Zhou S, Chang SS, McFate T, Verma A, Califano JA et al. (2009) Mitochondrial mutations contribute to HIFlalpha accumulation via increased reactive oxygen species and up-regulated pyruvate dehydrogenease kinase 2 in head and neck squamous cell carcinoma. Clin Cancer Res 15:476-484.

15. Ericson NG, Kulawiec M, Vermulst M, Sheahan K, O'Sullivan J, et al. (2012) Decreased mitochondrial DNA mutagenesis in human colorectal cancer. PLoS Genet 8: e1002689.

16. Dasgupta S, Hoque MO, Upadhyay S, Sidransky D (2008) Mitochondrial cytochrome $\mathrm{B}$ gene mutation promotes tumor growth in bladder cancer. Cancer Res 68: 700-706. 
17. Fliss MS, Usadel H, Caballero OL, Wu L, Buta MR, et al. (2000) Facile detection of mitochondrial DNA mutations in tumors and bodily fluids. Science 287: 2017-2019.

18. Lundholm K, Edström S, Karlberg I, Ekman L, Scherstén T (1982) Glucose turnover, gluconeogenesis from glycerol, and estimation of net glucose cycling in cancer patients. Cancer 50: 1142-1150.

19. Mazurek S, Boschek CB, Eigenbrodt E (1997) The role of phosphometabolites in cell proliferation, energy metabolism, and tumor therapy. J BioenergBiomembr 29: 315-330.

20. Ockner RK, Kaikaus RM, Bass NM (1993) Fatty-acid metabolism and the pathogenesis of hepatocellular carcinoma: review and hypothesis. Hepatology 18: 669-676.

21. Fischer CP, Bode BP, Souba WW (1998) Adaptive alterations in cellular metabolism with malignant transformation. Ann Surg 227: 627-634.

22. Bai RK, Leal SM, Covarrubias D, Liu A, Wong LJ (2007) Mitochondrial genetic background modifies breast cancer risk. Cancer Res 67: 4687-4694.

23. Putignani L, Raffa S, Pescosolido R, Rizza T, Del Chierico F, et al. (2012) Preliminary evidences on mitochondrial injury and impaired oxidative metabolism in breast cancer. Mitochondrion 12: 363-369.

24. Lee HC, Lu CY, Fahn HJ, Wei YH (1998) Aging- and smoking-associated alteration in the relative content of mitochondrial DNA in human lung. FEBS Lett 441: 292-296.

25. Renis M, Cantatore P, LoguercioPolosa P, Fracasso F, Gadaleta MN (1989) Content of mitochondrial DNA and of three mitochondrial RNAs in developing and adult rat cerebellum. J Neurochem 52: 750-754.

26. Verma M, Naviaux RK, Tanaka M, Kumar D, Franceschi C, et al. (2007) Meeting report: mitochondrial DNA and cancer epidemiology. Cancer Res 67: 437-439.

27. Shen J, Platek M, Mahasneh A, Ambrosone CB, Zhao H (2010) Mitochondrial copy number and risk of breast cancer: a pilot study. Mitochondrion 10: 62-68.

28. Yu M, Zhou Y, Shi Y, Ning L, Yang Y, et al. (2007) Reduced mitochondrial DNA copy number is correlated with tumor progression and prognosis in Chinese breast cancer patients. IUBMB Life 59: 450-457.

29. Tseng LM, Yin PH, Chi CW, Hsu CY, Wu CW, et al. (2006) Mitochondrial DNA mutations and mitochondrial DNA depletion in breast cancer. Genes Chromosomes Cancer 45: 629-638.

30. Bai RK, Chang J, Yeh KT, Lou MA, Lu JF, et al. (2011) Mitochondrial DNA content varies with pathological characteristics of breast cancer. J Oncol 2011: 496189.

31. Robin ED, Wong R (1988) Mitochondrial DNA molecules and virtual number of mitochondria per cell in mammalian cells. J Cell Physiol 136: 507-513.

32. Ng SB, Buckingham KJ, Lee C, Bigham AW, Tabor HK, et al. (2010) Exome sequencing identifies the cause of a mendelian disorder. Nat Genet 42: 30-35.

33. 1000 Genomes Project Consortium, Abecasis GR, Altshuler D, Auton A Brooks LD, et al. (2010) A map of human genome variation from population-scale sequencing. Nature 467: 1061-1073.

34. He Y, Wu J, Dressman DC, Iacobuzio-Donahue C, Markowitz SD, et al. (2010) Heteroplasmic mitochondrial DNA mutations in normal and tumour cells. Nature 464: 610-614.

35. Sondheimer N, Glatz CE, Tirone JE, Deardorff MA, Krieger AM, et al. (2011) Neutral mitochondrial heteroplasmy and the influence of aging. Hum Mol Genet 20: 1653-1659.

36. Smigrodzki RM, Khan SM (2005) Mitochondrial microheteroplasmy and a theory of aging and age-related disease. Rejuvenation Res 8: 172-198.

37. Kann LM, Rosenblum EB, Rand DM (1998) Aging, mating, and the evolution of mtDNAheteroplasmy in Drosophila melanogaster. ProcNatlAcadSci U S A 95: 2372-2377.

38. Schmitt MW, Kennedy SR, Salk JJ, Fox EJ, Hiatt JB, et al. (2012) Detection of ultra-rare mutations by next-generation sequencing. ProcNatlAcadSci U S A 109: 14508-14513.
39. Samuels DC, Han L, Li J, Quanghu S, Clark TA, et al. (2013) Finding the lost treasures in exome sequencing data. Trends Genet 29: 593-599.

40. Larman TC, DePalma SR, Hadjipanayis AG; Cancer Genome Atlas Research Network, Protopopov A, Zhang J, et al. (2012) Spectrum of somatic mitochondrial mutations in five cancers. ProcNatlAcadSci U S A 109: 14087-14091.

41. Picardi E, Pesole G (2012) Mitochondrial genomes gleaned from human whole-exome sequencing. Nat Methods 9: 523-524.

42. Tang S, Huang $\mathrm{T}$ (2010) Characterization of mitochondrial DNA heteroplasmy using a parallel sequencing system. Biotechniques 48: 287-296.

43. Guo Y CQ, Samuels DC, Ye F, Long J, Li CI, Winther JF, et al. (2012) The use of next generation sequencing technology to study the effect of radiation therapy on mitochondrial DNA mutation. Mutation Research Genetic Toxicology and Environmental Mutagenesis.

44. Goto H, Dickins B, Afgan E, Paul IM, Taylor J, et al. (2011) Dynamics of mitochondrial heteroplasmy in three families investigated via a repeatable re-sequencing study. Genome Biol 12: R59.

45. Guo Y, Cai Q, Samuels DC, Ye F, Long J, et al. (2012) The use of next generation sequencing technology to study the effect of radiation therapy on mitochondrial DNA mutation. Mutat Res 744: 154-160.

46. Ameur A, Stewart JB, Freyer C, Hagström E, Ingman M, et al. (2011) Ultra-deep sequencing of mouse mitochondrial DNA: mutational patterns and their origins. PLoS Genet 7: e1002028.

47. Guo Y, Li CI2, Sheng Q3, Winther JF4, Cai Q5, et al. (2013) Very lowlevel heteroplasmymtDNA variations are inherited in humans. J Genet Genomics 40: 607-615.

48. Cancer Genome Atlas Network (2012) Comprehensive molecular portraits of human breast tumours. Nature 490: 61-70.

49. Hazkani-Covo E, Zeller RM, Martin W (2010) Molecular poltergeists: mitochondrial DNA copies (numts) in sequenced nuclear genomes. PLoS Genet 6: e1000834.

50. Li M, Schroeder R, Ko A, Stoneking M (2012) Fidelity of captureenrichment for mtDNA genome sequencing: influence of NUMTs. Nucleic Acids Res 40: e137.

51. Li H, Handsaker B, Wysoker A, Fennell T, Ruan J, et al. (2009) The Sequence Alignment/Map format and SAMtools. Bioinformatics 25: 2078-2079.

52. Langmead B, Salzberg SL (2012) Fast gapped-read alignment with Bowtie 2. Nat Methods 9: 357-359.

53. Guo Y, Li J, Li CI, Shyr Y, Samuels DC (2013) MitoSeek: extracting mitochondria information and performing high-throughput mitochondria sequencing analysis. Bioinformatics 29: 1210-1211.

54. Bhat HK, Epelboym I (2004) Quantitative analysis of total mitochondrial DNA: competitive polymerase chain reaction versus real-time polymerase chain reaction. J BiochemMolToxicol 18: 180-186.

55. Castle JC, Biery M, Bouzek H, Xie T, Chen R, et al. (2010) DNA copy number, including telomeres and mitochondria, assayed using nextgeneration sequencing. BMC Genomics 11: 244.

56. Fliss MS, Usadel H, Caballero OL, Wu L, Buta MR, et al. (2000) Facile detection of mitochondrial DNA mutations in tumors and bodily fluids. Science 287: 2017-2019.

57. Wardell TM, Ferguson E, Chinnery PF (2003) Changes in the human mitochondrial genome after treatment of malignant disease. Mutation Research/Fundamental and Molecular Mechanisms of Mutagenesis 525:19-27.

58. Matsuyama W, Nakagawa M, Wakimoto J, Hirotsu Y, Kawabata M, et al. (2003) Mitochondrial DNA mutation correlates with stage progression and prognosis in non-small cell lung cancer. Hum Mutat 21: 441-443.

59. Lièvre A, Chapusot C, Bouvier AM, Zinzindohoué F, Piard F, et al. (2005) Clinical value of mitochondrial mutations in colorectal cancer. J ClinOncol 23: 3517-3525.

60. Kirches E (2009) Mitochondrial and nuclear genes of mitochondrial components in cancer. Curr Genomics 10: 281-293. 
Citation: Ye F, David C, Samuels, Guo Y (2014) Detection of Mitochondrial Mutations in Cancer by Next Generation Sequencing. Next Generat Sequenc \& Applic 1: 102. doi:10.4172/2469-9853.1000102

Page 5 of 5

61. Dinwiddie DL, Smith LD, Miller NA, Atherton AM, Farrow EG, et al. (2013) Diagnosis of mitochondrial disorders by concomitant next- generation sequencing of the exome and mitochondrial genome. Genomics 102: 148-156. 